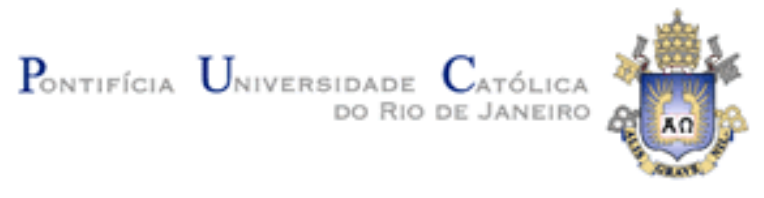

Felipe Roitman Rothschild

\title{
Credit and Income: Identification through Bank Competition
}

Dissertation presented to the Postgraduate Program in Economics of the Departamento de Economia, PUC-Rio as partial fulfillment of the requirements for the degree of Mestre em Economia

Advisor: Prof. João Manoel Pinho de Mello

Rio de Janeiro

Março de 2010 


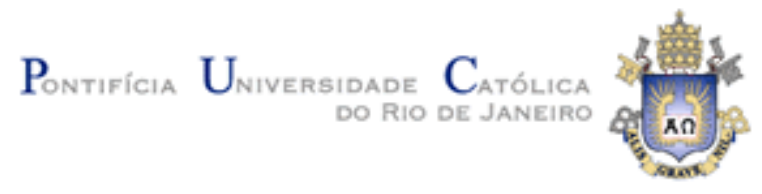

Felipe Roitman Rothschild

\section{Credit and Income: Identification through Bank Competition}

Dissertation presented to the Postgraduate Program in Economics of the Departamento de Economia, PUC-Rio as partial fulfillment of the requirements for the degree of Mestre em Economia. Approved by the following commission:

Prof. João Manoel Pinho de Mello Advisor Departamento de Economia - PUC-Rio

Prof. Claudio Abramovay Ferraz do Amaral Departamento de Economia - PUC-Rio

Prof. Tito Cordella

Banco Mundial

Profa. Mônica Herz

Coordenador Setorial do Centro de Ciências Sociais - PUC-Rio

Rio de Janeiro, 18 de Março de 2010 
All rights reserved.

\section{Felipe Roitman Rothschild}

Graduated in Economics from Insper - Instituto de Ensino e Pesquisa in 2007.

\section{Bibliographic Data}

\section{Rothschild, Felipe Roitman}

Credit and Income: Identification through Bank Competition / Felipe Roitman Rothschild; advisor: João Manoel Pinho de Mello. - Rio de Janeiro : PUC-Rio, Departamento de Economia, 2010.

$$
60 \text { f. : il. ; } 30 \mathrm{~cm}
$$

Dissertação (Mestrado em Economia)-Pontifícia Universidade Católica do Rio de Janeiro, Rio de Janeiro, 2010.

Inclui bibliografia

1. Economia - Teses. 2. Crédito. 3. Crescimento econômico. 4. Setor bancário. 5. Estrutura de mercado. I. Mello, João Manoel Pinho de. II. Pontifícia Universidade Católica do Rio de Janeiro. Departamento de Economia. III. Título. 
Aos dias 28, 29 e 30 de Janeiro de 2009, os dias mais valiosos de todo o mestrado. 


\section{Acknowledgments}

Aos meus pais, Eugênia e Charles, que sempre me apoiaram e incentivaram que eu levasse a vida da minha maneira.

Às minhas irmãs, Tatiana e Marina, que brincaram, apoiaram e fizeram de tudo para estar comigo nessa jornada chamada mestrado. Ao meu cunhado, Eduardo, por estar lá e ser um grande amigo. Ao meu sobrinho, Beni, por existir e tudo aquilo que representa para mim.

Às minhas eternas companheiras, Clarissa e Livia, por todos aqueles inúmeros momentos de diversão, alegria e aleatoriedade que fizeram com que meu último ano fosse o melhor possível.

Aos meus amigos, Werther e Victor, que viveram comigo essa jornada desde o começo: desde estudar para a Anpec até a defesa da dissertação. Pelos momentos que passamos juntos.

A todos aqueles grandes amigos que fiz durante toda essa jornada: Augusto, Bernard, Caruso, Henrique, Júlia, Lichand, Lucas, Magri, Marcão, Maurício, Paulo Vitor, Priscila e Theo. Pelos churrascos, tradição e comentários.

Ao meu orientador, João Manoel, que sugeriu, incentivou e me mostrou como intuir a economia por trás da situação e não a situação por trás da economia. Pelas sugestões, perguntas e oportunidades que me apresentou. Aos membros da minha banca, Tito e Cláudio, pelas sugestões e comentários que tanto acrescentaram na elaboração dessa tese. E ao Christiano pela ajuda com a base de dados.

E por fim, à Capes e ao Banco Opportunity pelos auxílios que permitiram que essa dissertação fosse realizada.

Essa dissertação não teria saído do papel se não fosse por cada uma dessas pessoas. 


\section{Resumo}

Rothschild, Felipe Roitman; De Mello, João Manoel Pinho. Crédito e Renda: Identificação via Concorrência Bancária. Rio de Janeiro, 2010. 60p. Dissertação de Mestrado - Departamento de Economia, Pontifícia Universidade Católica do Rio de Janeiro.

Os impactos do aprofundamento financeiro e crescimento econômico são amplamente discutidos na literatura econômica. Tendo em vista a recente crise financeira, seus efeitos de longo-prazo devem ser analisados mais detalhadamente. Esse trabalho busca investigar o mecanismo de transmissão do aumento de crédito via a estrutura concorrencial do mercado bancário. Com suficiente concorrência nesse setor, o número de bancos será um bom instrumento para crédito em uma regressão de crescimento. São encontradas evidências de variação de concorrência no mercado bancário brasileiro no nível local, bem como uma não linearidade na entrada de bancos em um determinado mercado. Por fim, ao incorporar essa relação não linear no número de bancos no primeiro estágio da regressão de crescimento, constata-se que, como esperado, não só os impactos da oferta de crédito sobre o crescimento econômico são positivos, mas também que a estratégia de identificação estava bem especificada, já que os efeitos positivos do crescimento sobre o estoque de crédito foram eliminados corretamente.

\section{Palavras-chave}

Crédito; Crescimento Econômico; Setor Bancário; Estrutura de Mercado. 


\section{Abstract}

Rothschild, Felipe Roitman; De Mello, João Manoel Pinho (Advisor). Credit and Income: Identification through Bank Competition. Rio de Janeiro, 2010. 60p. MSc Dissertation - Departamento de Economia, Pontifícia Universidade Católica do Rio de Janeiro.

The impacts of financial deepening on economic growth have been broadly discussed in the economic literature. Due to the recent financial crisis, its long-run effects must be carefully analyzed. This paper seeks to investigate the mechanism in which credit increases affect growth through the banking sector market structure. With enough competition in this market, the number of banks will be a good instrument for credit in a growth regression. Evidences of competition variability in the Brazilian banking sector are found at the local level as well as a non-linearity in bank entry at a specific market. Finally, after including this nonlinear relationship in the growth regression's first stage, it was found that, as expected, not only are the impacts of a credit increase positive but also that the identification strategy was well specified, since the positive effect of growth on credit was eliminated correctly.

\section{Keywords}

Credit; Economic Growth; Banking; Market Structure. 


\section{Contents}

1 Introduction 11

2 Identification Strategy 18

3 The Bresnahan and Reiss Model $\quad 21$

3.1. Econometric Specification 22

3.1.1. Entry-Threshold per firm Calculation 24

4 Brazilian Credit Market Evolution $\quad 25$

$\begin{array}{ll}5 \text { Data and Descriptive Statistics } & 27\end{array}$

6 Results $\quad 29$

6.1. Banking Industry Competitiveness 29

6.1.1 Basic Specifications 29

6.1.2. Results for the model with Variable Profits shocks 34

$\begin{array}{ll}\text { 6.1.3. Regional Results } & 37\end{array}$

6.1.4. Private Banks Competition $\quad 40$

6.2. Growth Regression $\quad 43$

6.2.1. $1^{\text {st }}$ Stage Results $\quad 44$

6.2.2. $2^{\text {nd }}$ Stage Results $\quad 46$

$\begin{array}{ll}\text { 6.2.3. Robustness: Conditional Convergence } & 48\end{array}$

$\begin{array}{lr}\text { 6.2.4. Transmission Mechanism } & 50\end{array}$

6.2.5. Alternative Competition Non-Linearity Specification 52

7 Concluding Remarks $\quad 56$

8 References $\quad 58$ 


\section{List of Tables}

Table 1 Descriptive Statistics - Bresnahan and Reiss 28

Table 2 Descriptive Statistics - Growth Regression 28

Table 3 Estimates of the Model's Parameters 30

Table 4 Entry-Thresholds per firm (in $\mathrm{R} \$$ thousands) and ratios for all three different specifications 32

Table 5 Market Competition Variability Test for all three specifications 33

Table 6 Estimates of the Heteroscedastic Model's Parameters 35

Table 7 Entry-Thresholds per firm (in $R \$$ thousands) and ratios from the heteroscedastic model 36

Table 8 Market Competition Variability Test for the Heteroscedastic Model 37

Table 9 Regional Parameter Estimates under specification (3) 38

Table 10 Regional Entry-Thresholds per firm (in $\mathrm{R} \$$ thousands) and ratios 39

Table 11 Regional Market Competition Variability Test 40

Table 12 Private Bank Model estimates for all three specifications 42

Table 13 Private Banks Entry Threshold per firm (in $\mathrm{R} \$$ thousands) and Competition Variability Test 43

Table 14 Parametric and Non-Parametric $1^{\text {st }}$ Stage Estimates 45

Table 15 Growth Regression Estimates $\quad 47$

Table 16 Growth Regression Estimates with convergence control $\quad 49$

Table 17 Financial Deepening Impact on different industries $\quad 50$

Table 18 Growth Regression with industry controls 51

Table $191^{\text {st }}$ stage results with dummies for the number of banks $\quad 54$

Table 20 2nd Stage Results with dummies for the number of banks 55 


\section{List of Figures}

Figure 1 Competition Variability 